\title{
WEIBULL MULTIPLICATIVE MODEL AND MACHINE LEARNING MODELS FOR FULL-AUTOMATIC DARK-SPOT DETECTION FROM SAR IMAGES
}

\author{
A. Taravat ${ }^{\mathrm{a}, *}$, F. Del Frate ${ }^{\mathrm{a}}$ \\ ${ }^{a}$ EO Lab, Dept. of Civil Engineering and Computer Science, University of Rome "Tor Vergata", Via del \\ Politecnico 1 \\ 00133 Rome, Italy - art23130@gmail.com
}

KEY WORDS: Weibull Multiplicative Model, Pulsed Coupled Neural Networks, Multilayer Perceptron Neural Networks, Dark Spot Detection, SAR Image Processing.

\begin{abstract}
:
As a major aspect of marine pollution, oil release into the sea has serious biological and environmental impacts. Among remote sensing systems (which is a tool that offers a non-destructive investigation method), synthetic aperture radar (SAR) can provide valuable synoptic information about the position and size of the oil spill due to its wide area coverage and day/night, and allweather capabilities. In this paper we present a new automated method for oil-spill monitoring. A new approach is based on the combination of Weibull Multiplicative Model and machine learning techniques to differentiate between dark spots and the background. First, the filter created based on Weibull Multiplicative Model is applied to each sub-image. Second, the sub-image is segmented by two different neural networks techniques (Pulsed Coupled Neural Networks and Multilayer Perceptron Neural Networks). As the last step, a very simple filtering process is used to eliminate the false targets. The proposed approaches were tested on 20 ENVISAT and ERS2 images which contained dark spots. The same parameters were used in all tests. For the overall dataset, the average accuracies of $94.05 \%$ and $95.20 \%$ were obtained for PCNN and MLP methods, respectively. The average computational time for dark-spot detection with a $256 \times 256$ image in about $4 \mathrm{~s}$ for PCNN segmentation using IDL software which is the fastest one in this field at present. Our experimental results demonstrate that the proposed approach is very fast, robust and effective. The proposed approach can be applied to the future spaceborne SAR images
\end{abstract}

\section{INTRODUCTION}

Accurate detection and forecast of hydrocarbon pollution in a timely manner would be beneficial to resource management for monitoring the marine environment. It is one of the most important applications for operational oceanography. Among remote sensing systems (which is a tool that offers a nondestructive investigation method), synthetic aperture radar (SAR) can provide valuable synoptic information about the position and size of the oil spill due to its wide area coverage and day/night, and all-weather capabilities (Taravat et al., 2013). Oil spills detection from SAR images divides into three steps: (1) Dark feature detection, (2) Physical feature extraction, and (3) Accurate discrimination between oil spills and lookalikes (such as internal waves, natural organics, algae and rain cells) (Solberg et al., 2007).

The extraction of the dark spots in SAR images is the prior step towards oil spill detection which is the most time consuming of the three steps. On the other hand, the accuracy of the further steps greatly rely on the accuracy of the extraction step. Aside from the accuracy of the segmentation results, one of the most significant parameters for evaluating the performance in this context is the processing time which is necessary to provide the segmented image.

In this paper we present a new fast, robust and effective automated method for oil-spill monitoring. A new approach from the combination of Weibull Multiplicative Model (WMM) and Neural Network models (Pulse Coupled Neural Networks (PCNN) and Multilayer Perceptron Neural Networks (MLP)) is proposed for achieving this goal.

The idea is to separate the detection process into two main steps, WMM enhancement and PCNN or MLP segmentation. The paper is organized in four sections. Section 2 contains a description of the models (WMM, PCNN, and MLP). In Section 3, a description of the experimental results obtained using ENVISAT, ERS2 images are analysed and explained. Section 4 contains the conclusion.

\section{METHODS}

Dark spot detection by the proposed approach can be grouped into two phases: First, the filter created based on weibull multiplicative model is applied to each sub-image which 
contains dark spots. Second, the sub-images are segmented by neural networks models (PCNN or MLP) (Brekke and Solberg, 2005; Taravat et al., 2013). As the last step, a very simple filtering process is used to eliminate the false targets.

\subsection{Weibull Multiplicative Model (WMM)}

The first step of dark feature detection is applying a filter which is used for removing image speckles and smoothing the image values. Traditionally, it has been assumed that the real and the imaginary parts of the received wave follow Gaussian distribution (Fernandes, 2001; Kuruoglu and Zerubia, 2004; Taravat et al., 2013). Another popular model is the Weibull distribution which has shown high degree of success in modeling urban scenes and sea clutter. WMM applies a nonlinear transformation to generate the texture image from the original image (Fernandes, 1998).

The Weibull-distributed random variable $\mathrm{x}$ with form parameter $\gamma_{x}>0$ and scale parameter $\beta_{x}>0$, has a probability density function given by:

$$
f(x)=\frac{\gamma_{x}}{\beta_{x}}\left(\frac{x}{\beta_{x}}\right)^{\gamma_{x}-1} \exp \left[-\left(\frac{x}{\beta_{x}}\right)^{\gamma_{x}}\right]
$$

The m-order moment can be expressed as,

$$
E\left[x^{m}\right]=m \beta_{x}^{m} \quad \Gamma\left(\mathrm{m} / \gamma_{x}\right) / \gamma_{x}
$$

For $\gamma_{x}=2$, the Weibull distribution becomes a Rayleigh distribution, for $\gamma_{x}=1$, it becomes an exponential distribution. It can be shown that $x^{a}$ with a $>0$ is also Weibull distributed. If, $\mathrm{z}=x^{a}$ with form and scale parameters given by, $\gamma_{z}=\gamma_{x} / a$ and $\beta_{z}=\beta_{x}^{a}$ follows that,

$$
f(z)=\frac{\gamma_{z}}{\beta_{z}}\left(\frac{z}{\beta_{z}}\right)^{\gamma_{z}-1} \exp \left[-\left(\frac{z}{\beta_{z}}\right)^{\gamma_{z}}\right]
$$

Consider $\mathrm{b}$, with $\mathrm{a}>\mathrm{b}>0$ in such a way that:

$$
\mathrm{z}=x^{a}=x^{b} x^{a-b}=\frac{x^{b}}{E\left[x^{b}\right]} E\left[x^{b}\right] x^{a-b}=s t
$$

Where (s) is the speckle, with unitary mean and $(\mathrm{t})$ is the texture of the Weibull-distributed variable $(\mathrm{z}) . \mathrm{z}$ is the variable for the SAR image.

$$
\mathrm{s}=x^{b} / E\left[x^{b}\right] \quad \mathrm{t}=x^{a-b} E\left[x^{b}\right]
$$

In this form, it is possible to express $\mathrm{z}$ as a multiplication of $\mathrm{s}$ by $t$, where $s$ is the speckle and $t$ is the texture of the Weibulldistributed variable $z$. The texture $t$ has Weibull distribution with form and scale parameter given, respectively, by:

$$
\gamma_{t}=\gamma_{x} /(a-b) \quad \beta_{t}=\beta_{x}^{a-b} E\left[x^{b}\right]
$$

and the speckle has Weibull distribution with form and scale parameter given, respectively, by:

$$
\gamma_{s}=\gamma_{x} / b \quad \beta_{s}=\beta_{x}^{b} / E\left[x^{b}\right]
$$

Let

$p=b / a, 0 \leq \mathrm{p}<1$

Then

$$
\mathrm{t}=x^{a-b} E\left[x^{b}\right]=x^{a(p-1)} E\left[x^{a p}\right]=z^{(1-p)} E\left[z^{p}\right]
$$

Using p-order moment equation $\mathrm{E}\left[z^{p}\right]$,

$$
t=p \beta_{z}^{p} \quad \Gamma\left(\mathrm{p} / \gamma_{z}\right) \mathrm{z}^{1-p} / \gamma_{z}
$$

Where $t$ can be considered as the filtered image and the factor 0 $\leq \mathrm{p}<1$ gives the filtering intensity. $\mathrm{p}$ value close to one makes a $\approx \mathrm{b}$ and the texture $\mathrm{t}$ will be constant (high filtering) and if $\mathrm{p}$ is close to zero then $\mathrm{a}>>\mathrm{b}$ and $\mathrm{t} \approx \mathrm{z}$ (low filtering).

\subsection{Pulse Coupled Neural Networks (PCNNs)}

Pulsed Coupled Neural Networks have been used for different fields (object extractions, edge detection, and texture analysis) of image processing (Del Frate et al., 2012; Lindblad and Kinser, 2005; Wang et al., 2010). The PCNN is a single layer, two-dimensional, laterally connected network of integrate-andfire neurons, with a 1:1 correspondence between the image pixels and network neurons (Taravat et al., 2013). The PCNN is categorized in the unsupervised neural networks group so it does not need any training stage (Del Frate et al., 2012; Lindblad and Kinser, 2005).

The input compartment composed by both the feeding and the linking inputs can be defined by the following expressions:

$$
\begin{gathered}
F_{i j}[n]=e^{-\alpha_{F}} \cdot F_{i j}[n-1]+S_{i j}+V_{F} \sum_{k l} M_{i j k l} Y_{k l}[n-1] \\
L_{i j}[n]=e^{-\alpha_{L}} \cdot L_{i j}[n-1]+V_{L} \sum_{k l} W_{i j k l} Y_{k l}[n-1]
\end{gathered}
$$

Where $S_{i j}$ is the input stimulus which is equal to the value of the pixel (ij). The compartment keeps memory of the previous state through the terms $F_{i j}[n-1]$ and $L_{i j}[n-1]$, which both of them decay in time by the exponent terms $\alpha_{F}$ and $\alpha_{L}$. The state of the feeding and linking inputs are combined into the linking compartment to create the internal state of the neuron "U" which is controlled by the linking strength, $\beta$ (Taravat et al., 2013). The internal activity is given by:

$$
U_{i j}[n]=F_{i j}[n]\left\{1+\beta L_{i j}[n]\right\}
$$

In the pulse generator compartment, the internal state of the neuron is compared to a dynamic threshold, $\Theta$, to produce the output, Y, by:

$$
Y_{i j}[n]= \begin{cases}1, & \text { If } U_{i j}[n]>\theta_{i j}[n] \\ 0, & \text { Otherwise }\end{cases}
$$


The threshold mechanism is described as:

$$
\theta_{i j}[n]=e^{-\alpha \theta} \cdot \theta_{i j}[n-1]+V_{\theta} Y_{i j}[n]
$$

The parameter $\mathrm{V}_{\theta}$ is necessary to adjust the effect of the neuron state to prevent the saturation and also to control the thresholding value. The term $\alpha_{\theta}$ adjust the level of decreasing the threshold value during the iteration process (Taravat et al., 2013).

\subsection{Multilayer Perceptron Neural Networks segmentation}

A neuron $\mathrm{k}$ can be described by writing the following pair of equations:

$$
\begin{gathered}
u_{k}=\sum_{i=1}^{n} w_{k i} x_{i} \\
y_{k}=\varphi\left(u_{k}+b_{k}\right)(x+a)^{n}=\sum_{k=0}^{n}\left(\begin{array}{l}
n \\
k
\end{array}\right) x^{k} a^{n-k}
\end{gathered}
$$

Where $x_{1}, \ldots \ldots \ldots, x_{n}$ are the input signals, $w_{k 1}, \ldots \ldots \ldots, w_{k n}$ are the synaptic weights of neuron $\mathrm{k}, u_{k}$ is the linear combiner output due to the input signals, $b_{k}$ is the bias, $\varphi($.$) is the activation function, and y_{k}$ is the output signal of the neuron. Logistic function defines by the following equation where $\mathrm{a}>0$ is the slope parameter:

$$
\varphi(v)=\frac{1}{1+e^{-a v}}
$$

Typically, MLPs consists of the input layer, one or more hidden layers of computation nodes, and an output layer of computation nodes (Del Frate et al., 2000; Taravat and Del Frate, 2012).

IDL and the neural network simulator (SNNS) developed at the University of Stuttgart, Germany, has been used for developing WMM model and classification algorithm implementation, respectively (Zell A. et al., 1995). Both versions (Adaptive and Non-adaptive Weibull Multiplicative Model) can be obtained in IDL from Geoinformation Online (Taravat, 2013).

\section{RESULTS AND DISCUSSION}

The models have been tested on a dataset of ENVISAT-ASAR and ERS2-SAR images. Radiometric calibration has been applied to the dataset in order to generate a backscatter $\left(\sigma^{0}\right)$ image. The test dataset contains 20 images with $256 \times 256$ pixels. We applied WMM approach to all 20 test images using the filtering intensity $\mathrm{P}=0.7$ and a $3 \times 3$ kernel size (kernel sizes of $5 \times 5,7 \times 7$ and $9 \times 9$ blur the image) surrounding the pixel to be filtered.

In MLP model the number of units in the hidden layer and the training/testing phase settings represent the fundamental tasks. The pixels for train/test the net are 1200 pixels which extracted from different types of dark spot and different sea status. The training sets contain $60 \%$ and the test sets contain $40 \%$ of all pixels which are not belonging to the training sets.

Pixel selection for train/test set has been done randomly and repeated four times. The topology 1-4-2 has been finally chosen for its good performance in terms of classification accuracy and RMSE. The number of about 11,000 training cycles was sufficient to get the network learned. One MLP NNs has been used for classifying all images.

For the PCNN model a unique best setting has been obtained for both ENVISAT and ERS products, using parameter's values as follow: $\alpha_{\mathrm{L}}=0.2, \alpha_{\mathrm{F}}=2, \alpha_{\theta}=2, \mathrm{~V}_{\mathrm{L}}=0.6, \mathrm{~V}_{\mathrm{F}}=0.8, \mathrm{~V}_{\theta}=1.5$ and $\beta=0.5$. The $3 \times 3$ square matrixes of synaptic weights $\mathrm{M}$ and $\mathrm{W}$ are defined with a linking radius $\mathrm{r}=1.5$. After segmentation phase, the area less than 20 pixels omit from the image to eliminate the regions which incorrectly detected as dark spots.

For accuracy assessment, from each sub-image 250 pixels have randomly been selected and then labelling made by visual interpretation. Figure 1 and Figure 2 shows two sample test images from different types of dark spots and different sea status (a not well-defined massive dark spot located in a very heterogeneous background and a not well-defined linear dark spot within a homogeneous background).

The accuracy of the test dataset segmented by MLP is $95.20 \%$ with a standard deviation of 1.6 whereas the accuracy of the same dataset segmented by PCNN method is $94.05 \%$ with a standard deviation of 2.1. Dark-spot detection by the PCNN model with a $256 \times 256$ image can be completed in about 4 seconds on a pc with an Intel Pentium dual-core, a speed of 2.2 $\mathrm{GHz}$ and a RAM memory of $2.00 \mathrm{~GB}$ which is rather competitive with respect to the existing methods in the literature.



Fig.1. Results of the proposed approach on a not well-defined massive dark spot located in a very heterogeneous background (the boundary between the dark feature and the surrounding water is not well defined). (a) Original SAR images after preprocessing. (b) WMM Filtering with $\mathrm{P}=0.7$, window $3 \times 3$. (c) PCNN result. (d) MLP result. 


\section{CONCLUSION}

In the present study a detailed research on the ability of using WMM \& MLP \& PCNN model for dark-spot detection in SAR imagery demonstrated. To test the performance of the proposed approaches, we applied it to a dataset containing 20 ENVISAT, ERS2 images which cover all potential anomaly cases.

The average accuracy for the overall dataset segmented by PCNN was $94.05 \%$ and the average computational time for a detection window was 4 seconds using IDL software whereas the average accuracy for the overall dataset segmented by MLP was $95.20 \%$. Results showed that MLP has higher performance in the situations where PCNN generates poor accuracy.

Overall, the results demonstrate that the proposed approaches for dark-spot detection is effective, fast and robust. The proposed approach can be applied to the future spaceborne $\mathrm{C}$ band SAR with some parameters adjustment based on the type of data.



Fig.2. Results of the proposed approach on a not well-defined linear dark spot within a homogeneous background. (a) Original SAR images after pre-processing. (b) WMM Filtering with $\mathrm{P}=0.7$, window $3 \times 3$. (c) MLP result. (Fourth $\mathrm{Col}$ ) PCNN result.

\section{ACKNOWLEDGEMENTS}

The authors would like to thank Prof. D. Fernandes, Head of the Telecommunications Department, Centro Técnico de Aeronáutica (CTA) - Instituto Tecnológico de Aeronáutica (ITA), Brazil, for his valuable cooperation and helpful suggestions. Data were provided by the European Space Agency on Cat-1 proposal numbers 1418 and 13926.

\section{REFERENCES}

Brekke, C., Solberg, A.H.S., 2005. Oil spill detection by satellite remote sensing. Remote Sens Environ 95, 1-13.
Del Frate, F., Latini, D., Pratola, C., Palazzo, F., 2012. PCNN for automatic segmentation and information extraction from $\mathrm{X}$ band SAR imagery. International Journal of Image and Data Fusion, 1-14.

Del Frate, F., Petrocchi, A., Lichtenegger, J., Calabresi, G., 2000. Neural networks for oil spill detection using ERS-SAR data. Ieee Transactions on Geoscience and Remote Sensing 38, 2282-2287.

Fernandes, D., 1998. Segmentation of SAR Images with Weibull Distribution, Proceedings of the IGARSS.

Fernandes, D., 2001. Weibul Texture Filter (WTF) for SAR images, Proceedings of the IGARSS.

Kuruoglu, E.E., Zerubia, J., 2004. Modeling SAR images with a generalization of the Rayleigh distribution. Ieee $\mathrm{T}$ Image Process 13, 527-533.

Lindblad, T., Kinser, J.M., 2005. Image Processing Using Pulse-Coupled Neural Networks. Berlin, Heidelberg : Springer Verlag Berlin Heidelberg

Solberg, A.H.S., Brekke, C., Husoy, P.O., 2007. Oil spill detection in Radarsat and Envisat SAR images. Ieee Transactions on Geoscience and Remote Sensing 45, 746-755.

Taravat, A., 2013. Adaptive \& Non-adaptive Weibull Multiplicative filter for SAR images. http://www.geoinformation.com.

Taravat, A., Del Frate, F., 2012. Development of band ratioing algorithms and neural networks to detection of oil spills using Landsat ETM+ data. EURASIP Journal on Advances in Signal Processing 2012, 107.

Taravat, A., Latini, D., Del Frate, F., 2013. Fully Automatic Dark-Spot Detection From SAR Imagery With the Combination of Nonadaptive Weibull Multiplicative Model and PulseCoupled Neural Networks. IEEE Transactions on Geoscience and Remote Sensing 52.

Wang, Z., Ma, Y., Cheng, F., Yang, L., 2010. Review of pulsecoupled neural networks. Image and Vision Computing 28, 513.

Zell A., Mamier G., Michael Vogt, Niels Mache, Ralf Hubner, S.D., Kai-Uwe Herrmann, Tobias Soyez, Michael Schmalzl, Tilman Sommer, Artemis Hatzigeorgiou, Dietmar Posselt, Tobias Schreiner, Bernward Kett, Gianfranco Clemente, Jens Wiel, Jurgen Gatter, 1995. SNNS Stuttgart neural network simulator user manual. Inst. Parallel Distributed High Performance Syst., Univ. Stuttgart, Stuttgart, Germany. 